\title{
Données nouvelles sur la néphrotoxicité de l'uranium
}

\author{
Y. GUEGUEN*, C. ROUAS
}

(Manuscrit reçu le 5 janvier 2012, accepté le 5 mars 2012)

RÉSUMÉ L'uranium est un radioélément ainsi qu'un métal lourd auquel l'homme peut être exposé du fait de sa présence dans l'environnement ou des activités humaines. Il exerce principalement une toxicité chimique au niveau du tubule contourné proximal du rein après exposition aiguë ou chronique. Après exposition à une forte dose d'uranium, la néphrite tubulaire aiguë est révélée par une protéinurie ainsi qu'une diminution du débit de filtration glomérulaire. Plus récemment, l'utilisation de marqueurs d'intégrité tissulaire telle que la béta 2-microglobuline ou des enzymes tissulaires a pu être corrélée aux altérations histopathologiques des tubules contournés proximaux. Lors d'exposition chronique à de faible niveau, l'utilisation de marqueurs plus spécifiques et plus sensibles s'avère nécessaire lorsque les lésions sont faibles. Des études expérimentales développées au laboratoire ont montré la pertinence de certains de ces biomarqueurs tel que Kim-1 pour détecter de faibles altérations rénales. L'uranium exerce sa cytotoxicité au niveau des cellules épithéliales des tubules contournés proximaux probablement du fait de son entrée dans la cellule où il s'accumule préférentiellement dans le noyau cellulaire, lorsqu'il n'est pas sous forme de précipités. Les mécanismes conduisant à la mort des cellules ne sont pas encore totalement élucidés mais le stress oxydant semble y jouer un rôle important.

ABSTRACT New data on uranium nephrotoxicity.

Uranium is an element and also a heavy metal to which humans can be exposed due to its natural presence or human activities. It has mainly a chemical toxicity on the proximal convoluted tubules of the kidney after acute or chronic exposure. At high dose exposure, acute tubular nephritis is observed, as indicated by proteinuria and a decreased glomerular filtration rate. More recently, the use of structural biomarkers such as beta 2-microglobuline or tubular enzymes has been correlated with histopathological injury of the proximal convoluted tubules. During chronic exposure at a low level, the use of some of these sensitive and specific biomarkers would be necessary, particularly when the injury is slight. Recent experimental studies in the laboratory have shown the relevance of such new biomarkers as Kim1 to detect slight renal injury. Uranium exerts its toxicity on epithelial tubular cells in which it accumulates mainly in the nucleus when it is not precipitated. The mechanisms leading to cell death are not totally elucidated but oxidative stress seems to play an important role.

Keywords: uranium / kidney / toxicity / exposure / nephrotoxicity

Institut de radioprotection et de sûreté nucléaire (IRSN), PRP-HOM, SRBE, LRTOX, Laboratoire de radiotoxicologie expérimentale, BP 17, 92262 Fontenay-aux-Roses, France.

* e-mail: yann.gueguen@irsn.fr 


\section{Introduction}

L'uranium est un composé chimique qui possède des propriétés radioactives. Ce radioélément naturel (UNat) est présent de façon ubiquitaire dans le sol, l'eau et l'air. Les activités humaines d'enrichissement en isotope 235 de l'uranium à des fins civiles et militaires aboutissent à la formation d'uranium enrichi (UE) et appauvri (UA). Pour l'UNat et l'UA, auxquels la population générale peut être exposée par ingestion du fait de leurs présences dans l'environnement, la toxicité chimique est prépondérante (Craft et al., 2004 ; Sztajnkrycer et Otten, 2004) et s'exerce principalement au niveau des reins. À l'inverse, la toxicité radiologique de l'uranium devient plus importante pour les formes enrichies en isotope 235 (supérieure à $3 \%$ ); elle concerne uniquement les travailleurs du nucléaire et se manifeste au niveau des poumons, des os, des reins et du foie selon les voies d'entrées et la transférabilité du composé (Filippova et al., 1978 ; Morris et al., 1989).

La biocinétique de l'uranium dépend de sa voie d'entrée (inhalation, ingestion, blessure) et de sa solubilité (Karpas et al., 1998 ; Leggett et Harrison, 1995 ; Wrenn et al., 1985). Quelque que soit la voie d'exposition, l'uranium se distribue via la circulation sanguine dans tous les tissus et il s'accumule préférentiellement dans le rein et l'os (La Touche et al., 1987 ; Leggett et Pellmar, 2003 ; Paquet et al., 2006).

Du fait de son rôle épurateur, le rein est l'organe principal d'exposition aux effets toxiques de nombreux métaux lourds (dont l'uranium). En effet, l'uranium est filtré à travers la membrane glomérulaire et passe au niveau du tubule contourné proximal (TCP). Il se déposerait ensuite principalement à la surface des cellules tubulaires rénales, au niveau cortical et au niveau de la région juxtaglomérulaire (Paquet et al., 2004). À ce niveau, l'uranium serait réabsorbé par les cellules épithéliales de la bordure en brosse. En conséquence, en cas d'intoxication à l'U, la néphrotoxicité est le symptôme prépondérant.

L'objectif de cette revue est donc de dresser un tableau des connaissances actuelles sur la néphrotoxicité de l'uranium qui sont essentiellement basée sur sa toxicité à forte dose. Néanmoins, le manque de connaissance sur la néphrotoxicité à faible dose et à long terme de l'uranium a été complété plus récemment, notamment par de nouvelles études développées au sein de notre laboratoire. Nous détaillerons ensuite les principaux mécanismes cellulaires sous-jacents qui ont pu être identifiés à ce jour permettant une meilleure compréhension du processus d'atteinte et l'identification de nouveaux marqueurs. 


\section{Néphrotoxicité due à une exposition aiguë}

La dose létale 50 (DL50) de l'uranium (dose qui induit $50 \%$ de mortalité des animaux après exposition unique) est estimée à $204 \mathrm{mg} / \mathrm{kg}$ chez le rat après exposition orale unique par gavage (Domingo et al., 1987 ; Voegtlin et Hodge, 1953). L'exposition aiguë à de fortes doses d'uranium se manifeste chez le rongeur par une altération de l'état général de l'animal. Même si de nombreux troubles apparaissent (perte de poids, hypothermie, tremblements, hémorragies oculaires et nasales, myosis, exophtalmie), le décès des animaux est attribué à la toxicité rénale causée par une néphrite tubulaire aiguë (Albina et al., 2005 ; Blantz, 1975). Les premiers signes de la toxicité tissulaire de l'uranium sont décrits au niveau du rein, l'organe de stockage à court terme (Diamond et al., 1989 ; Haley, 1982 ; Leggett, 1989).

\subsection{Physiopathologie (marqueurs plasmatiques et urinaires)}

Biochimiquement, un certain nombre de marqueurs témoignent de l'atteinte organique et fonctionnelle des reins (Tab. I).

Plusieurs études ont rapporté une augmentation plasmatique de deux marqueurs communément utilisés mais peu discriminatifs des lésions rénales (la créatinine et l'urée) en cas d'exposition aiguë à l'uranium (par voie intramusculaire ou sous cutanée) (Banday et al., 2008b ; Blantz, 1975 ; Fukuda et al., 2006 ; Gueguen et al., 2006 ; Kobayashi et al., 1984). En parallèle, il a été décrit chez le rongeur, une diminution de la clairance de la créatinine (indicatif d'une diminution du taux de filtration glomérulaire) (Banday et al., 2008b ; Haley, 1982). Deux mécanismes peuvent alors être envisagés : l'un lié à une atteinte tubulaire et à la fuite des solutés absorbés à travers la membrane tubulaire endommagée et l'autre à une atteinte structurale glomérulaire.

Conformément à cette atteinte tubulaire, l'augmentation de la concentration urinaire d'enzymes révélatrices de lésions tissulaires ( $\mathrm{N}$-acétylglucosaminidase, phosphatase alkaline, gamma glutamyl transpeptidase) a largement été décrite (Banday et al., 2008b ; Diamond et al., 1989 ; Fukuda et al., 2006) ainsi que l'augmentation d'excrétion d'électrolytes, de protéines telles que de béta2microglobuline qui sont des signes d'altérations tubulaires fonctionnelles (Banday et al., 2008b ; Fukuda et al., 2006 ; Haley, 1982).

Enfin, des modifications chez le rat du taux de la forme active de la vitamine D (jouant un rôle crucial dans l'homéostasie phosphocalcique) et des enzymes impliquées dans son métabolisme au niveau rénal ont été observées en cas d'exposition aiguë à l'UA (injection intra-gastrique, $204 \mathrm{mg} / \mathrm{kg}$ ) ce qui témoigne d'une atteinte des activités métaboliques du rein (Tissandie et al., 2006). 
TABLEAU I

Résumé des effets rénaux relevés lors d'études expérimentales chez l'animal après exposition aiguë à l'uranium.

Summary of acute exposure toxicity studies in experimental animals exposed to uranium on kidney.

\begin{tabular}{|c|c|c|c|c|}
\hline Références & Uranium & Espèces & $\begin{array}{l}\text { Conditions } \\
\text { d'expositions }\end{array}$ & Observations \\
\hline Zimmerman et al. (2007) & uranyl nitrate & Rat & ip 0,1 to $1 \mathrm{mg} / \mathrm{kg}$ & $\begin{array}{l}\boldsymbol{\lambda} \text { de la créatinine, urée et albumine } \\
\text { à forte dose }\end{array}$ \\
\hline Nomiyama et al. (1974) & uranyl acetate & Lapin & iv $0,2 \mathrm{mg} / \mathrm{kg}$ & $\begin{array}{l}\boldsymbol{\lambda} \text { glucose, } \mathrm{LDH} \text {, phosphatase } \\
\text { alcaline urinaire }\end{array}$ \\
\hline Banday et al. (2008b) & uranyl nitrate & Rat & ip $0,5 \mathrm{mg} / \mathrm{kg}$ & $\begin{array}{l}\boldsymbol{\lambda} \text { de la créatinine, urée, cholestérol } \\
\text { et phospholipides }\end{array}$ \\
\hline Diamond et al. (1989) & $\mathrm{UO} 2 \mathrm{~F} 2$ & Rat & ip $0,66 \mathrm{mg} / \mathrm{kg}$ & $\begin{array}{l}\text { Y du poids corporel et des reins } \\
\text { त de LDH, AST, protéinurie, } \\
\text { albuminurie }\end{array}$ \\
\hline Fukuda et al. (2006) & uranyl nitrate & Rat & im 0,2 to $2 \mathrm{mg} / \mathrm{kg}$ & $\begin{array}{l}\text { y ALT, AST, protéines } \\
\text { त urée, créatinine, Phosphatase } \\
\text { alcaline }\end{array}$ \\
\hline Kobayashi et al. (1984) & uranyl acetate & Lapin & iv $2 \mathrm{mg} / \mathrm{kg}$ & $\boldsymbol{y}$ DFG et débit urinaire \\
\hline Sanchez et al. (2001) & uranyl nitrate & Rat & ip 2,5 à $5 \mathrm{mg} / \mathrm{kg}$ & $\begin{array}{l}\boldsymbol{\lambda} \text { débit urinaire, protéines, NAG } \\
\text { urinaires } \\
\boldsymbol{У} \text { DFG }\end{array}$ \\
\hline Taulan et al. (2006) & uranyl nitrate & Souris & ip $5 \mathrm{mg} / \mathrm{kg}$ & $\begin{array}{l}\lambda \text { GT et glucose urinaire, créatinine } \\
\text { et urée plasmatique }\end{array}$ \\
\hline Kato et al. (1994) & uranyl acetate & Rat & iv $5 \mathrm{mg} / \mathrm{kg}$ & $\begin{array}{l}\boldsymbol{\lambda} \text { créatinine, urée, activité rénine } \\
\text { et excrétion de } \mathrm{Na} \\
\boldsymbol{У} \text { poids corporel }\end{array}$ \\
\hline Domingo et al. (1987) & uranyl acetate & Rat & $\mathrm{sc} 10 \mathrm{mg} / \mathrm{kg}$ & $\begin{array}{l}\boldsymbol{\lambda} \text { protéinurie } \\
\mathbf{y} \text { créatinine urinaire } \\
\boldsymbol{\lambda} \text { ALT, AST, urée, créatinine }\end{array}$ \\
\hline Haley et al. (1982) & uranyl nitrate & Rat & ip $10 \mathrm{mg} / \mathrm{kg}$ & $\begin{array}{l}\boldsymbol{\lambda} \text { Na et protéines urinaires } \\
\text { et } \boldsymbol{\Downarrow} \text { DFG }\end{array}$ \\
\hline Gueguen et al. (2006) & uranyl nitrate & Rat & ip $11,5 \mathrm{mg} / \mathrm{kg}$ & $\boldsymbol{\lambda}$ créatinine, urée, ALT, AST \\
\hline Blantz (1975) & uranyl nitrate & Rat & iv $25 \mathrm{mg} / \mathrm{kg}$ & $\begin{array}{l}\text { У DFG et taux de filtration du } \\
\text { néphron }\end{array}$ \\
\hline Fukuda et al. (2005) & uranyl nitrate & Rat & $\operatorname{im~} 7,9$ to $126 \mathrm{mg} / \mathrm{kg}$ & $\begin{array}{l}\text { У poids corporel et mort après } 3 \\
\text { à } 7 \text { jours }\end{array}$ \\
\hline
\end{tabular}

\subsection{Atteinte histologique}

Chez le rongeur, que l'exposition soit due à une injection sous-cutanée $(10 \mathrm{mg} / \mathrm{kg}$ de nitrate d'uranyle) ou intra-péritonéale $(0,66 \mathrm{ou} 1,32 \mathrm{mg} / \mathrm{kg}$ de fluorure d'uranyle), les lésions rénales touchent préférentiellement les deux tiers distaux du tube contourné proximal (TCP) (Diamond et al., 1989 ; Haley et al., 1982). Cette 
zone est décrite comme étant la zone la plus active du néphron en termes d'activité de réabsorption/sécrétion et d'activité métabolique, d'où les désordres d'équilibre électrolytique ou de synthèse d'hormone décrits précédemment en cas d'atteinte.

Histologiquement, l'uranium induit une perte focale de la bordure en brosse ainsi qu'une induction de vacuoles du TCP dès la première heure consécutive à l'injection sous-cutanée de nitrate d'uranyle (10 mg/kg) (Haley, 1982). Chez le rat, cinq jours après le traitement, l'apparition de foyers nécrotiques est notée. Ce type d'atteinte est observé chez le rongeur mais aussi chez le lapin traité par injection intraveineuse d'acétate d'uranyle (2 mg/kg) (Kobayashi et al., 1984). À titre indicatif, la concentration minimale induisant une atteinte histologique rénale est observée entre 0,7 et $1,4 \mu \mathrm{g}$ d'uranium par gramme de rein (soit 0,66 à $1,32 \mathrm{mg} / \mathrm{kg}$ de poids corporel) chez le rat (Diamond et al., 1989 ; Leggett, 1989).

Bien que la majorité des effets concerne l'atteinte du tubule proximal, l'atteinte glomérulaire a également été décrite lorsque la dose administrée est importante $(\sim 10 \mathrm{mg} / \mathrm{kg})$ (Haley, 1982). Après contamination au fluorure d'uranium, l'anse de Henlé ainsi que les tubes collecteurs sont également affectés (Diamond et al., 1989); l'intensité des lésions varie donc en fonction de la dose d'uranium administrée.

Le caractère réversible de la lésion tubulaire est montré à travers différents modèles lors d'une exposition relativement faible et pour lesquels un arrêt de l'exposition a été étudié (Diamond et al., 1989 ; Haley et al., 1982 ; Kobayashi et al., 1984). Malgré une régénération tubulaire, une fibrose interstitielle et une infiltration de cellules mononuclées indiquent des conséquences chroniques de l'atteinte rénale (Haley et al., 1982).

\section{Néphrotoxicité due à une exposition chronique}

\subsection{Physiopathologie (marqueurs plasmatiques et urinaires) cf. 1.1}

Les données (expérimentales et épidémiologiques) propres à l'exposition chronique à l'U (UA ou Unat) sont plus rares que celles à une exposition aiguë (Tab. II). On peut néanmoins en retenir qu' une exposition chronique ne se résume pas à une somme d'expositions aiguës, ce qui ouvre de nouvelles pistes de recherche.

Le taux plasmatique de créatinine est jusqu'à présent le biomarqueur le plus utilisé pour évaluer un dysfonctionnement rénal et ce malgré sa faible spécificité. Expérimentalement, après contamination chronique par voie orale $(1$ à $600 \mathrm{mg} / \mathrm{L})$, différents paramètres urinaires (l'urée, le glucose, les protéines et l'albumine ainsi 
TABLEAU II

Résumé des effets rénaux relevés lors d'études expérimentales chez l'animal après exposition chronique à l'uranium.

Summary of chronic exposure toxicity studies in experimental animals exposed to uranium on kidney.

\begin{tabular}{|c|c|c|c|c|}
\hline Références & Uranium & Espèces & $\begin{array}{l}\text { Conditions } \\
\text { d'expositions }\end{array}$ & Observations \\
\hline Gilman et al. (1998b) & $\begin{array}{l}\text { uranyl } \\
\text { nitrate }\end{array}$ & rat & $\begin{array}{l}\text { Oral } 0,02 \text { to } 400 \mathrm{mg} / \mathrm{kg} \\
\text { pendant } 3 \text { mois }\end{array}$ & $\begin{array}{l}\text { У } \mathrm{Hb}, \text { Er, glucose et poids corporel } \\
\text { non corrélés à la dose } \\
\text { Lésions histopathologiques dés la } \\
\text { plus faible concentration }\end{array}$ \\
\hline Tissandie et al. (2007) & $\begin{array}{l}\text { uranyl } \\
\text { nitrate }\end{array}$ & rat & $\begin{array}{l}\text { Oral } 2,7 \mathrm{mg} / \mathrm{kg} \text { pendant } \\
9 \text { mois }\end{array}$ & $\begin{array}{l}\text { Y de la vitamine } \mathrm{D} \text { plasmatique et des } \\
\text { gènes associés }\end{array}$ \\
\hline Gueguen et al. (2007) & $\begin{array}{l}\text { uranyl } \\
\text { nitrate }\end{array}$ & rat & $\begin{array}{l}\text { Oral } 2,7 \mathrm{mg} / \mathrm{kg} \text {, } \\
\text { pendant } 9 \mathrm{mois}\end{array}$ & $\begin{array}{l}\text { Après traitement unique au } \\
\text { paracétamol, } \boldsymbol{\lambda} \text { de la nécrose des TCP }\end{array}$ \\
\hline Ortega et al. (1989) & $\begin{array}{l}\text { uranyl } \\
\text { nitrate }\end{array}$ & rat & Oral 2 à $16 \mathrm{mg} / \mathrm{kg}$ & $\boldsymbol{\lambda}$ de la glycémie quelque soit la dose \\
\hline Taulan et al. (2004) & $\begin{array}{l}\text { uranyl } \\
\text { nitrate }\end{array}$ & souris & Oral 13 à 26 mg/kg & $\begin{array}{l}\boldsymbol{y} \text { urée et } \boldsymbol{\lambda} \text { créatinine plasmatique; } \\
\boldsymbol{y} \text { poids des reins }\end{array}$ \\
\hline Gilman et al. (1998a) & $\begin{array}{l}\text { uranyl } \\
\text { nitrate }\end{array}$ & lapin & $\begin{array}{l}\text { Oral } 0,02 \text { à } 400 \mathrm{mg} / \mathrm{kg} \\
\text { pendant } 3 \text { mois }\end{array}$ & $\begin{array}{l}\text { Pas de modification biochimique } \\
\text { Lésions histopathologiques pour la } \\
\text { plus forte concentration }\end{array}$ \\
\hline Berradi et al. (2008) & $\begin{array}{l}\text { uranyl } \\
\text { nitrate }\end{array}$ & rat & $\begin{array}{l}\text { Oral } 2,7 \mathrm{mg} / \mathrm{kg} \text {, } \\
\text { pendant } 9 \mathrm{mois}\end{array}$ & $\boldsymbol{y}$ taux sanguin de globules rouges \\
\hline Zhu et al. (2008) & $\begin{array}{l}\text { uranyl } \\
\text { nitrate }\end{array}$ & rat & $\begin{array}{l}\text { Implant } 200 \text { à } 600 \\
\mathrm{mg} / \mathrm{kg} \text { pendant } 3 \text { à } 12 \\
\text { mois }\end{array}$ & $\begin{array}{l}\boldsymbol{\lambda} \text { urée, créatinine plasmatiques, } \\
\boldsymbol{\lambda} \text { beta2-microglobuline et albumine } \\
\text { urinaire }\end{array}$ \\
\hline $\begin{array}{l}\text { Maynard et Hodge } \\
\text { (1953) }\end{array}$ & $\begin{array}{l}\text { uranyl } \\
\text { nitrate }\end{array}$ & rat & $\begin{array}{l}\text { Oral } 4 \text { à } 200 \mathrm{mg} / \mathrm{kg} \\
\text { pendant } 1 \text { à } 24 \mathrm{mois}\end{array}$ & $\begin{array}{l}\boldsymbol{\lambda} \text { lésions histologiques pour la dose } \\
\text { la plus forte } \\
\boldsymbol{\lambda} \text { créatinine et urée plasmatique, } \\
\text { protéinurie }\end{array}$ \\
\hline Stokinger et al. (1953) & $\begin{array}{l}\text { uranyl } \\
\text { fluoride }\end{array}$ & chien & $\begin{array}{l}\text { Inhalation } 0,05 \mathrm{mg} / \mathrm{m}^{3} \\
\text { pendant } 12 \text { à } 24 \mathrm{mois}\end{array}$ & $\begin{array}{l}\boldsymbol{\lambda} \text { catalase } \\
\text { y DFG }\end{array}$ \\
\hline
\end{tabular}

que les activités de LDH ou de NAG) ont été mesurés. L'hyperglucosurie semble être l'effet le plus persistant alors que les autres paramètres étudiés ne varient pas ou peu (Gilman et al., 1998a ; Ortega et al., 1989). A contrario, après exposition chronique via la mise en place d'implants sous-cutanés d'uranium, une augmentation des taux plasmatiques d'urée, de créatinine, d'albumine et de $\beta 2$ microglobuline est observée (Zhu et al., 2008). L'utilisation de nouveaux biomarqueurs d'atteinte rénale plus sensibles et plus spécifiques (Kim-1 (kidney injury molecule), ostéopontine, kallikrein) a été étudiée très récemment et montre qu'une exposition chronique à faible dose n'aggrave pas les effets néphrotoxiques de certains médicaments comme la gentamicine (Rouas et al., 2011). Dans cette étude, Kim-1, reconnu comme l'un des marqueurs les plus prometteurs pour le suivi d'atteintes rénales aiguë ou chronique (Vaidya et al., 2009 ; Gueguen et al., 2012), voit son taux tissulaire et urinaire augmenter proportionnellement à l'atteinte histologique induite par la gentamicine, mais n'est pas accru chez les 
animaux contaminés par l'uranium. Tout comme lors d'une exposition aiguë, des perturbations fonctionnelles peuvent également être observées telles que sur le métabolisme rénal de la vitamine D (Tissandie et al., 2007) ou du fer (Berradi et al., 2008).

Chez l'Homme, des études épidémiologiques ont été menées sur des populations consommant de l'eau de puits dont la teneur en uranium est supérieure aux normes OMS ( $>30 \mu \mathrm{g} / \mathrm{L})(\mathrm{WHO}, 2011)$. L'augmentation de l'excrétion urinaire du glucose ainsi que l'augmentation urinaire de la phosphatase alcaline et de la $\beta 2$-microglobuline témoignent d'une atteinte tubulaire potentielle chez les populations exposées (Zamora et al., 1998, 2009). Une augmentation réversible de la $\beta 2$-microglobuline a également été rapportée chez le plus jeune enfant d'une famille exposé à l'uranium via l'eau de puits (entre 866 et $1160 \mu \mathrm{g} / \mathrm{L}$ ) soulignant la sensibilité accrue des jeunes enfants aux expositions environnementales (Magdo et al., 2007). Une autre étude menée sur une population de 325 personnes exposées à l'eau contenant de l'uranium (moyenne d'exposition $28 \mu \mathrm{g} / \mathrm{L}$ ) semble indiquer une corrélation entre la concentration d'uranium dans l'eau, celle retrouvée dans les urines et une augmentation de l'excrétion urinaire du calcium et du phosphate (Kurttio et al., 2002). Ces effets n'ont cependant pas été retrouvés dans des travaux postérieurs menés par la même équipe sur la même cohorte (Kurttio et al., 2006). Les études de Selden montrent également l'absence de variation de certains marqueurs de néphrotoxicité (dont la $\beta 2$-microglobuline) malgré l'exposition de la population étudiée à des concentrations d'uranium allant jusqu'à $470 \mu \mathrm{g} / \mathrm{L}$ (Selden et al., 2009).

Par ailleurs, bien qu'une étude conclue en l'absence de contamination de l'organisme des militaires des suites de l'exposition aux obus (à tête d'uranium) durant les conflits du Golfe et des Balkans (Cazoulat et al., 2008) des études complémentaires ont montré la présence d'uranium dans les urines jusqu'à 18 ans après exposition à l'uranium (McDiarmid et al., 2011, 2000 ; Squibb and McDiarmid, 2006). Le suivi des vétérans souligne une tendance non significative à des dysfonctionnements tubulaires rénaux attribuables à l'uranium (McDiarmid et al., 2009, 2006).

\subsection{Modifications morphologiques}

Expérimentalement, en cas de contamination chronique (per os ou par des implants intra-musculaires), les atteintes principales sont décrites au niveau du TCP avec notamment l'apparition de vacuoles et de vésicules chez le rat (Gilman et al., 1998 ; Ortega et al., 1989 ; Zhu et al., 2009b) ou chez le lapin (Taylor et al., 1997) mais sans démonstration de relation dose-effet. Il a ainsi été montré une dégénérescence de l'épithélium associée à une perte focale de la bordure en brosse 
(Taylor et al., 1997 ; Zhu et al., 2009b). Des atteintes glomérulaires et de l'interstitium ont également été notées chez le rat (Gilman et al., 1998 ; Zhu et al., 2009b). Dans le cas d'une administration unique d'un médicament, le paracétamol, à une dose élevée une augmentation de la nécrose des cellules du TCP est observée chez les animaux exposés à une faible dose d'uranium par rapport aux animaux non-exposés (Gueguen et al., 2007).

\section{Mécanismes de néphrotoxicité de l'U}

Des études menées sur des cellules rénales d'espèces différentes (rat, cochon, homme) (Carriere et al., 2004 ; Mirto et al., 1999 ; Prat et al., 2005) ainsi que sur des cellules osseuses (Milgram et al., 2008), neuronales et hépatiques (Rouas et al., 2010) montrent que l'uranium possède des propriétés cytotoxiques. Néanmoins, les mécanismes exacts de la toxicité de l'uranium restent à l'heure actuelle méconnus. La relation entre l'entrée de l'uranium dans la cellule et sa toxicité a été étudiée sur des cellules du TCP (ex vivo et in vitro). Plusieurs auteurs proposent que la cytotoxicité de l'uranium est directement liée à son entrée cellulaire alors que d'autres le démentent (Leggett, 1989 ; Muller et al., 2006, Carriere et al., 2008 ; Ghadially et al., 1982 ; L'Azou et al., 2002 ; Mirto et al., 1999).

\subsection{Distribution cellulaire de l'U et toxicité}

Les techniques d'imagerie employées jusqu'à présent, de type microscopie à transmission électronique (MET) ou microscopie électronique à balayage (SEM), ont permis de mettre en évidence la présence de précipités d'uranium, anciennement appelées « uraniosomes » (Ghadially et al., 1982) aussi bien dans les espaces intracellulaires (cytoplasme dont lysosomes) qu'extracellulaires (Galle, 1974). D'après l'analyse aux rayons X, ces précipités seraient composés de potassium, de phosphate, de calcium et de soufre complexés à l'uranium (Carriere et al., 2008 ; Ghadially et al., 1982 ; Mirto et al., 1999 ; Muller et al., 2006).

Néanmoins, l'apparition de ces précipités est décrite pour des concentrations d'uranium élevées et supérieures aux concentrations minimales induisant une mort cellulaire. Globalement, la concentration qui induit $50 \%$ de mortalité pour des cellules rénales d'origine humaine est aux environs de $500 \mu \mathrm{M}$ d'uranium (Carriere et al., 2004 ; Prat et al., 2005 ; Rouas et al., 2010). Des variations de sensibilité à l'uranium ont été observées et seraient dues à la fois à la différence de robustesse des modèles cellulaires mais également à la spéciation du composé (Carriere et al., 2004 ; Milgram et al., 2008a).

Nous avons récemment proposé que la forme physique de l'uranium (soluble ou précipité) et sa localisation intra-cellulaire pouvaient jouer un rôle dans la 
toxicité cellulaire de l'uranium (Rouas et al., 2010). Dans cette étude, grâce à l'utilisation de la technique d'imagerie SIMS (Secondary Ion Mass Spectrometry) permettant une analyse élémentaire et isotopique, nous avons pu montrer la présence préférentielle d'uranium (soluble) dans le noyau des cellules exposés à de faibles concentrations d'uranium $(<50 \mu \mathrm{M})$. De plus, la présence de précipités intra-cytoplasmique d'uranium à une dose supérieure mais non-cytotoxique $(100 \mu \mathrm{M})$ montre que la viabilité cellulaire n'est pas uniquement corrélée à la présence de précipités (Fig. 1). En effet, à cette concentration non cytotoxique, l'uranium est à la fois présent sous forme soluble dans le noyau et sous forme précipitée dans le cytoplasme. Cette répartition hétérogène de l'uranium dans les cellules a également été observée récemment lors d'études in vivo chez des rats contaminés via l'eau de boisson (Tessier et al., 2012).
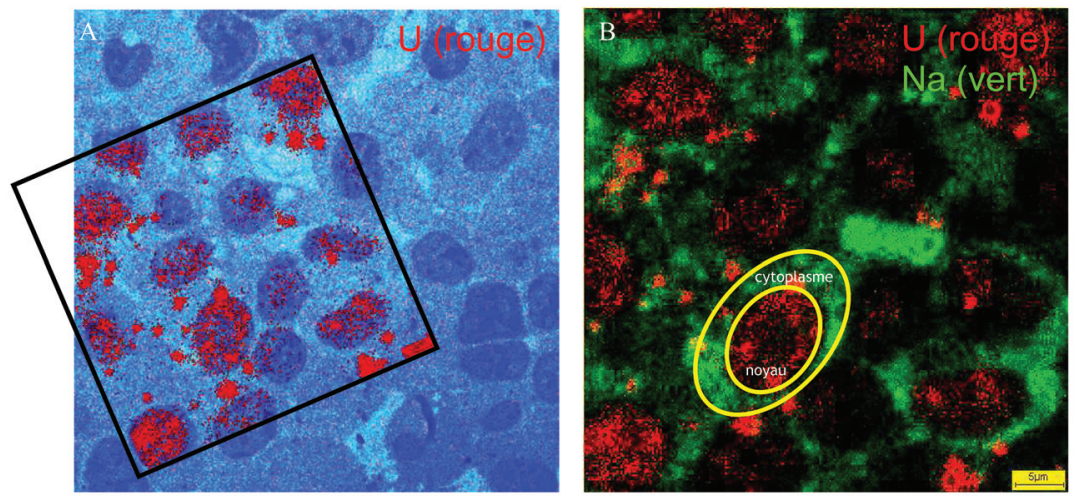

Figure 1 - Localisation cellulaire de l'uranium après exposition à l'uranium (100 $\mu M)$ de cellules rénales humaine (HEK293) pendant $24 \mathrm{~h}$ : (A) Superposition d'image histologique (coloration au bleu de toluidine) et image SIMS (uranium en rouge) (Secondary Ion Mass Spectrometry) obtenues sur coupe minces $(0,5 \mu \mathrm{m})$ de cellules incluent en résine Epoxy après $24 \mathrm{~h}$ d'exposition à l'uranium appauvri (100 $\mathrm{\mu M})$. (B) Superposition d'images ionique du $\mathrm{Na}^{+}$(vert) et ${ }^{238} \mathrm{U}^{+}$ (rouge) obtenues par analyse SIMS sur coupe minces $(0,5 \mu \mathrm{m})$ de cellules incluent en résine Epoxy après $24 \mathrm{~h}$ d'exposition à l'uranium appauvri $(100 \mu \mathrm{M})$. Ces résultats ont été présentés lors du dernier congrès de l'ICRR à Varsovie (28 août au $1^{\text {er }}$ septembre) et publiés précédemment en comparaison à d'autres types cellulaires (Rouas et al., 2010).

Uranium cellular localisation in human kidney cells (HEK293) exposed to uranium (100 $\mu$ M) during 24 h: (A) Superposition of optical microscope image (toluidine blue staining) and ionic image (U in red) obtained with SIMS (Secondary Ion Mass Spectrometry) technology after serial thin section $(0.5 \mu \mathrm{m})$ of cells embedded in Epon-type resin. (B) Superposition of Na+ (green) and $238 U+($ red) ionic images obtained with SIMS technology after serial thin section $(0.5 \mu \mathrm{m})$ of cells embedded in Epon-type resin. 
La présence de l'uranium dans le compartiment intra-cellulaire soulève donc la question de son mécanisme d'entrée. Plusieurs hypothèses sont actuellement émises. La première serait que l'uranium (soluble ou précipité) entre dans la cellule par endocytose puis se retrouve dans les vésicules lysosomales. Du fait de l'acidification du milieu environnant, l'uranium soluble précipiterait (Galle, 1997 ; Leggett, 1989). La seconde hypothèse est que l'uranium entrerait dans la cellule par l'intermédiaire de transporteurs. Le rôle d'un transport de l'uranium via les transporteurs de type NaPi-IIa (co-transporteur de phosphate dépendant du sodium) a été mis en évidence (Muller et al., 2006, 2008).

\subsection{Transporteurs d'électrolytes et solutés}

En plus de ce transporteur $\mathrm{NaPi}$, différentes études tant in vivo qu'in vitro ont rapporté au préalable des effets inhibiteurs de l'uranium sur les transports actifs d'ions sodium ou potassium ( $\mathrm{NaK}$ ATPase, Na ATPase) et autres processus consommateurs d'ATP et ce pour des concentrations supérieures à $1 \mathrm{mM}$ (Brady et al., 1989 ; Hori et al., 1985 ; Schwartz et Flamenbaum, 1976). Ainsi une diminution de la consommation d'ATP a été observée sur des cellules rénales en cultures exposées à l'uranium. Par ailleurs, le transport du glucose ainsi que son métabolisme au sein du TCP serait également affecté après exposition à l'uranium et en ferait un $4^{\mathrm{e}}$ transporteur altéré par l'uranium au niveau cellulaire (Goldman et al., 2006 ; Renault et al., 2010 ; Vicente-Vicente et al., 2010).

L'analogie structurale de l'uranium avec le calcium pourrait également être explorée plus avant quant aux perturbations du transport du calcium qui pourrait en découler. En effet, après exposition de cellules rénales à l'uranium, on observe une modification de l'expression de gènes tels que la calmoduline ou de protéines impliquées dans le transport intracellulaire du calcium (Prat et al., 2005).

\subsection{Stress oxydant}

Suite à une exposition à l'uranium, le stress oxydant est l'un des mécanismes les plus étudiés pour expliquer sa toxicité et notamment sa néphrotoxicité. Chez le rongeur, suite à une exposition aiguë, à raison de 0,5 à $5 \mathrm{mg} / \mathrm{kg}$ en injection intrapéritonéale unique, une variation au niveau rénal de l'expression des enzymes antioxydantes (SOD, catalase...) et une augmentation de la peroxydation lipidique sont observées (Banday et al., 2008a ; Belles et al., 2007 ; Priyamvada et al., 2010). Chez le rat, après exposition chronique ( 3 mois, $40 \mathrm{mg} / \mathrm{kg}$ ) l'uranium induit une augmentation de marqueurs du stress oxydant (peroxydation lipidique et oxydation du glutathion (GSSG)) au niveau rénal (Linares et al., 2006). In vitro, sur des cellules rénales en culture, une augmentation de la production d'espèces réactives de l'oxygène est également observée et impliquerait une entrée des 
cellules en apoptose à partir de concentrations élevées $(>600 \mu \mathrm{M})$ (Thiebault et al., 2007). Comme cela a été observé pour d'autres métaux, les perturbations de l'homéostasie redox sont donc susceptibles d'entrâner des phénomènes d'adaptation allant d'une induction de la réponse antioxydante à une mort cellulaire programmée (apoptose) afin de préserver le tissu environnant. Si ces déséquilibres ne peuvent être compensés, tels que lors d'expositions aiguës à de fortes doses de métaux lourds, des changements morphologiques et fonctionnels majeurs sont observés pouvant conduire à une nécrose cellulaire (Barbier et al., 2005 ; Valko et al., 2005).

\subsection{Signalisation cellulaire}

Une étude de transcriptomique menée sur des cellules HEK-293 (Human embryonic kidney) montre que $25 \%$ des gènes dont l'expression est modulée par l'exposition à de l'UA sont impliqués dans les voies de transduction et de trafic cellulaire, notamment dans celles du calcium (Prat et al., 2005).

Des travaux de toxico-génomique menés sur des souris exposées au nitrate d'uranyle soit par l'eau de boisson $(80 \mathrm{mg} / \mathrm{L})$ soit par injection intra-péritonéale $(5 \mathrm{mg} / \mathrm{kg})$ montrent des perturbations de l'expression génique de plus de 200 gènes dont certains impliqués dans le stress oxydant, la transduction des signaux cellulaires, le transport ou encore le métabolisme et le catabolisme cellulaire (Taulan et al., 2006, 2004).

L'altération du métabolisme cellulaire est en effet un facteur important de la toxicité cellulaire de l'uranium (Banday et al., 2008b ; Brady et al., 1989 ; Fleck et al., 2002) et peut être mis en relation avec les conséquences histologiques et fonctionnelles décrites précédemment.

\section{Conclusion}

L'uranium est un élément radioactif naturel qui serait modérément néphrotoxique à des doses environnementales comparativement à d'autres métaux lourds tels que le plomb, le cadmium ou le mercure. Du fait de sa présence dans l'environnement, iI pourrait engendrer des désordres rénaux que ce soit chez l'homme ou l'animal. Depuis les premiers travaux en 1949 étudiant la toxicologie ou la pharmacologie de l'uranium chez l'animal (Maynard et al., 1949) de nombreuses autres études notamment concernant les effets d'une exposition chronique à faible dose sont venues compléter l'étude déjà bien établie des effets d'une exposition aiguë. Néanmoins, quel que soit le type d'exposition, les mécanismes de cette toxicité rénale ne sont pas encore totalement élucidés et il n'existe toujours pas de marqueurs spécifiques d'une néphrotoxicité à l'uranium. Un certain nombre de 
nouveaux marqueurs plasmatiques et urinaires de la fonction et de l'intégrité des TCP (béta2-microglobuline, RBP...) ont toutefois été utilisés lors d'études expérimentales ou cliniques permettant une détection plus précise de l'atteinte rénale. Afin d'évaluer au mieux l'importance des effets observés selon les schémas d'exposition, les recherches futures tant expérimentales que cliniques ou épidémiologiques devront se poursuivre. Pour ce faire des études mécanistiques pourront apporter une aide précieuse pour la compréhension du processus d'atteinte rénale et l'identification de nouveaux marqueurs plus spécifiques.

Remerciements. Ces travaux font partie du programme de recherche ENVIRHOM soutenu par l'Institut de Radioprotection et de Sûreté Nucléaire. Les auteurs remercient $C$. Tessier et D. Suhard (LRC/IRSN) pour les analyses SIMS.

\section{RÉFÉRENCES}

Albina M.L. et al. (2005) Restraint stress does not enhance the uranium-induced developmental and behavioral effects in the offspring of uranium-exposed male rats, Toxicology 215, 69-79.

Banday A.A. et al. (2008a) Time dependent effects of gentamicin on the enzymes of carbohydrate metabolism, brush border membrane and oxidative stress in rat kidney tissues, Life Sci. 82, 450459.

Banday A.A. et al. (2008b) Effect of uranyl nitrate on enzymes of carbohydrate metabolism and brush border membrane in different kidney tissues, Food. Chem. Toxicol. 46, 2080-2088.

Barbier O. et al. (2005) Effect of heavy metals on, and handling by, the kidney, Nephron Physiol. 99 , 105-110.

Belles M. et al. (2007) Melatonin reduces uranium-induced nephrotoxicity in rats, J. Pineal. Res. 43, 87-95.

Berradi H. et al. (2008) Renal anemia induced by chronic ingestion of depleted uranium in rats, Toxicol. Sci. 103, 397-408.

Blantz R.C. (1975) The mechanism of acute renal failure after uranyl nitrate, J. Clin. Invest. 55, 621635.

Brady H.R. et al. (1989) Early effects of uranyl nitrate on respiration and $\mathrm{K}+$ transport in rabbit proximal tubule, Kidney Int. 36, 27-34.

Carriere M. et al. (2004) Influence of uranium speciation on normal rat kidney (NRK-52E) proximal cell cytotoxicity, Chem. Res. Toxicol. 17, 446-452.

Carriere M. et al. (2008) Transmission electron microscopic and X-ray absorption fine structure spectroscopic investigation of $U$ repartition and speciation after accumulation in renal cells, J. Biol. Inorg. Chem. 13, 655-662.

Cazoulat A. et al. (2008) Urinary uranium analysis results on Gulf war or Balkans conflict veterans, Pathol. Biol. (Paris) 56, 77-83.

Craft E. et al. (2004) Depleted and natural uranium: chemistry and toxicological effects, J. Toxicol. Environ. Health Part B Crit. Rev. 7, 297-317.

Diamond G.L. et al. (1989) Reversible uranyl fluoride nephrotoxicity in the Long Evans rat, Fundam. Appl. Toxicol. 13, 65-78.

Domingo J.L. et al. (1987) Acute toxicity of uranium in rats and mice, Bull. Environ. Contam. Toxicol. 39, 168-174. 


\section{DONNÉES NOUVELLES SUR LA NÉPHROTOXICITÉ DE L’URANIUM}

Filippova L.G. et al. (1978) Late sequelae of administering enriched uranium to rats, Radiobiologiia 18, 400-405.

Fleck M. et al. (2002) Renal interstitial fibrosis (RIF): II. Ultrasound follow up study of single uranyl nitrate administration causing renal dysfunction in rats - comparison with histologic and functional renal parameters, Exp. Toxicol. Pathol. 54, 15-23.

Fukuda S. et al. (2005) Toxicity of uranium and the removal effects of CBMIDA and EHBP in simulated wounds of rats, Health Phys. 89, 81-88.

Fukuda S. et al. (2006) Clinical diagnostic indicators of renal and bone damage in rats intramuscularly injected with depleted uranium, Radiat. Prot. Dosim. 118, 307-314.

Galle P. (1974) Role of lysosomes and mitochondria in the handling of mineral compounds by the kidney, J. Microsc. 19, 17-24.

Galle P. (1997) Uranium, Toxiques nucléaires, Masson (Ed.), Paris.

Ghadially F.N. et al. (1982) Uraniosomes produced in cultured rabbit kidney cells by uranyl acetate, Virchows Arch. B Cell Pathol. 39, 21-30.

Gilman A.P. et al. (1998a) Uranyl nitrate: 91-day exposure and recovery studies in the male New Zealand white rabbit, Toxicol. Sci. 41, 138-151.

Gilman A.P. et al. (1998b) Uranyl nitrate: 28-day and 91-day toxicity studies in the Sprague-Dawley rat, Toxicol. Sci. 41, 117-128.

Goldman M. et al. (2006) Nephrotoxicity of uranyl acetate: effect on rat kidney brush border membrane vesicles, Arch. Toxicol. 80, 387-393.

Gueguen Y. et al. (2006) Short-term hepatic effects of depleted uranium on xenobiotic and bile acid metabolizing cytochrome P450 enzymes in the rat, Arch. Toxicol. 80, 187-195.

Gueguen Y. et al. (2007) Effect of acetaminophen administration to rats chronically exposed to depleted uranium, Toxicology 229, 62-72.

Gueguen Y. et al. (2012) Les biomarqueurs d'atteinte rénale, Nephrol. Ther. 8 (3), 146-155.

Haley D.P. (1982) Morphologic changes in uranyl nitrate-induced acute renal failure in saline- and water-drinking rats, Lab. Invest. 46, 196-208.

Haley D.P. et al. (1982) The long-term effects of uranyl nitrate on the structure and function of the rat kidney, Virchows Arch. B Cell Pathol. 41, 181-192.

Hori R. et al. (1985) Transport of p-aminohippurate, tetraethylammonium and D-glucose in renal brush border membranes from rats with acute renal failure, J. Pharmacol. Exp. Ther. 233, 776-781.

Karpas Z. et al. (1998) Uptake of ingested uranium after low "acute intake", Health Phys. 74, 337-345.

Kato A. et al. (1994) Effects of oxygen free radical scavengers on uranium-induced acute renal failure in rats, Free Radic. Biol. Med. 16, 855-859.

Kobayashi S. et al. (1984) Glomerular alterations in uranyl acetate-induced acute renal failure in rabbits, Kidney Int. 26, 808-815.

Kurttio P. et al. (2002) Renal effects of uranium in drinking water, Environ. Health Perspect. 110, $337-$ 342.

Kurttio P. et al. (2006) Kidney toxicity of ingested uranium from drinking water, Am. J. Kidney Dis. 47, 972-982.

L'Azou B. et al. (2002) Effects of cadmium and uranium on some in vitro renal targets, Cell Biol. Toxicol. 18, 329-340.

La Touche Y.D. et al. (1987) Absorption and biokinetics of U in rats following an oral administration of uranyl nitrate solution, Health Phys. 53, 147-162.

Leggett R.W. (1989) The behavior and chemical toxicity of U in the kidney: a reassessment, Health Phys. 57, 365-383. 
Leggett R.W. et al. (1995) Fractional absorption of ingested uranium in humans, Health Phys. 68, 484498.

Leggett R.W. et al. (2003) The biokinetics of uranium migrating from embedded DU fragments, $J$. Environ. Radioact. 64, 205-225.

Linares V. et al. (2006) Assessment of the pro-oxidant activity of uranium in kidney and testis of rats, Toxicol. Lett. 167, 152-161.

Maynard E.A. et al. (1949) Studies of the toxicity of various uranium compounds when fed to experimental animals, The Pharmacology and Toxicology of Uranium Compounds. Division VI, Vol. I, (C. Voegtlin and H.C. Hodge, Eds), McGraw Hill, New York, NY, pp. 309-376.

Maynard E.A. et al. (1953) Oral toxicity of uranium compounds, The Pharmacology and Toxicology of Uranium Compounds. Division VI, Vol. I, (C. Voegtlin and H.C. Hodge, Eds), McGraw Hill, New York, NY, pp. 309-376.

McDiarmid M.A. et al. (2000) Health effects of depleted uranium on exposed Gulf War veterans, Environ. Res. 82, 168-180.

McDiarmid M.A. et al. (2011) Longitudinal health surveillance in a cohort of Gulf War veterans 18 years after first exposure to depleted uranium, J. Toxicol. Environ. Health Part A 74, 678-691.

Milgram S. et al. (2008) Cytotoxic and phenotypic effects of uranium and lead on osteoblastic cells are highly dependent on metal speciation, Toxicology 250, 62-69.

Mirto H. et al. (1999) Intracellular behaviour of uranium(VI) on renal epithelial cell in culture (LLCPK1): influence of uranium speciation, Toxicol. Lett. 104, 249-256.

Morris K.J. et al. (1989) Studies of alveolar cell morphometry and mass clearance in the rat lung following inhalation of an enriched uranium dioxide aerosol, Radiat. Environ. Biophys. 28, 141154.

Muller D. et al. (2006) Role of the sodium-dependent phosphate co-transporters and of the phosphate complexes of uranyl in the cytotoxicity of uranium in LLC-PK1 cells, Toxicol. Appl. Pharmacol. 214, 166-177.

Muller D.S. et al. (2008) Role of the sodium-dependent phosphate cotransporters and absorptive endocytosis in the uptake of low concentrations of uranium and its toxicity at higher concentrations in LLC-PK1 cells, Toxicol. Sci. 101, 254-262.

Nomiyama K. et al. (1974) Assay of urinary enzymes in toxic nephropathy, Toxicol. Appl. Pharmacol. 27, 484-490.

Ortega A. et al. (1989) Evaluation of the oral toxicity of uranium in a 4-week drinking-water study in rats, Bull. Environ. Contam. Toxicol. 42, 935-941.

Paquet F. et al. (2004) Speciation and internal dosimetry: from chemical species to dosimetric models, Spéciation et dosimétrie interne: des espèces chimiques aux modèles dosimétriques, Radioprotection 39, 3, 341-354.

Paquet F. et al. (2006) Accumulation and distribution of uranium in rats after chronic exposure by ingestion, Health Phys. 90, 139-147.

Prat O. et al. (2005) Transcriptomic and proteomic responses of human renal HEK293 cells to uranium toxicity, Proteomics 5, 297-306.

Priyamvada S. et al. (2010) Studies on the protective effect of dietary fish oil on uranyl-nitrate-induced nephrotoxicity and oxidative damage in rat kidney, Prostaglandins Leukot Essent Fat. Acids $\mathbf{8 2}$, 35-44.

Renault S. et al. (2010) Uranyl nitrate inhibits lactate gluconeogenesis in isolated human and mouse renal proximal tubules: a 13C-NMR study, Toxicol. Appl. Pharmacol. 242, 9-17.

Rouas C. et al. (2010) Distribution of Soluble Uranium in the Nuclear Cell Compartment at Subtoxic Concentrations, Chem. Res. Toxicol. 23, 1883-1889. 
Rouas C. et al. (2011) Effect of nephrotoxic treatment with gentamicin on rats chronically exposed to uranium, Toxicology 279, 27-35.

Sanchez D.J. et al. (2001) Nephrotoxicity of simultaneous exposure to mercury and uranium in comparison to individual effects of these metals in rats, Biol. Trace Elem. Res. 84, 139-154.

Schwartz J.H. et al. (1976) Uranyl nitrate and $\mathrm{HgCl} 2$-induced alterations in ion transport, Kidney Int. Suppl. 6, S123-127.

Squibb K.S. et al. (2006) Depleted uranium exposure and health effects in Gulf War veterans, Philos. Trans. R. Soc. Lond. B Biol. Sci. 361, 639-648.

Stokinger H.E. et al. (1953) Toxicity following inhalation for 1 and 2 years, The Pharmacology and Toxicology of Uranium Compounds. Division VI, Vol. I, (C. Voegtlin and H.C. Hodge, Eds), McGraw Hill, New York, NY, pp. 309-376.

Sztajnkrycer M.D. et al. (2004) Chemical and radiological toxicity of depleted uranium, Mil. Med. 169, 212-216.

Taulan M. et al. (2006) Comprehensive analysis of the renal transcriptional response to acute uranyl nitrate exposure, BMC Genomics 7, 2.

Taulan M. et al. (2004) Renal toxicogenomic response to chronic uranyl nitrate insult in mice, Environ. Health Perspect. 112, 1628-1635.

Taylor D.M. et al. (1997) Environmental uranium and human health, Rev. Environ. Health 12, 147-157.

Tessier C. et al. (2012) Uranium microdistribution in renal cortex of rats after chronic exposure: a study by secondary ion mass spectrometry microscopy, Microsc. Microanal. 18, 123-133.

Thiebault C. et al. (2007) Uranium induces apoptosis and is genotoxic to normal rat kidney (NRK-52E) proximal cells, Toxicol. Sci. 98, 479-487.

Tissandie E. et al. (2007) In vivo effects of chronic contamination with depleted uranium on vitamin $\mathrm{D}(3)$ metabolism in rat, Biochim. Biophys. Acta 1770, 266-272.

Vaidya V.S. et al. (2009) A rapid urine test for early detection of kidney injury, Kidney Int. 76, 108-114.

Valko M. et al. (2005) Metals, toxicity and oxidative stress, Curr. Med. Chem. 12, 1161-1208.

Vicente-Vicente L. et al. (2010) Nephrotoxicity of uranium: pathophysiological, diagnostic and therapeutic perspectives, Toxicol. Sci. 118, 324-347.

Voegtlin C. et al. (1953) Pharmacology and Toxicology of uranium compounds, Vol. 3-4, McGrawHill, New York

WHO (2011) Guidelines for drinking-water quality, fourth edition.

Wrenn M.E. et al. (1985) Metabolism of ingested U and Ra, Health Phys. 48, 601-633.

Zhu G. et al. (2009) Renal dysfunction induced by long-term exposure to depleted uranium in rats, Arch. Toxicol. 83 (1), 37-46.

Zimmerman K.L. et al. (2007) Temporal clinical chemistry and microscopic renal effects following acute uranyl acetate exposure, Toxicol. Pathol. 35, 1000-1009. 